\title{
Was the Narrow Line Seyfert 1 RGB J0044+193 ever radio loud?
}

\author{
T. J. Maccarone ${ }^{1}$, J. C. A. Miller-Jones ${ }^{1}$, R. P. Fender ${ }^{2,1}$, and G. G. Pooley ${ }^{3}$ \\ 1 Astronomical Institute "Anton Pannekoek," University of Amsterdam, Kruislaan 403, Amsterdam 1098 SJ, The Netherlands \\ e-mail: [tjm;jmiller]@science.uva.nl \\ 2 Department of Physics and Astronomy, University of Southampton, Hampshire SO17 1BJ, UK \\ e-mail: rpf@phys.soton.ac.uk \\ 3 Mullard Radio Astronomy Observatory, Cavendish Laboratory, Madingley Road, Cambridge CB3 OHE, UK
}

Received 4 November 2004 / Accepted 16 December 2004

\begin{abstract}
We show new radio data and a re-analysis of old data for the Narrow Line Seyfert 1 (NLSy1) galaxy RGB J0044+193. This galaxy has previously been suggested to be both radio loud, and highly variable in the radio. As most NLSy 1 galaxies are radio quiet, this was interpreted as possible evidence that this AGN's jet was beamed toward the observer. This object was probably never either radio loud and nor variable - the one exception to this rule is a marginal detection of the object in the 1987 Green Bank survey, which we argue may have been spurious.
\end{abstract}

Key words. accretion, accretion disks - galaxies: jets - quasars: individual: RGB J0044+193 - radio continuum: galaxies galaxies: nuclei - galaxies: active

\section{Introduction}

In recent years, a growing understanding of the coupling between accretion disks and relativistic jets has been developing. In particular, it has been found that the ratio of monochromatic radio flux density to accretion luminosity increases with black hole mass and decreases with increasing luminosity in Eddington units (see e.g. Boroson 2002; Gallo et al. 2003; Merloni et al. 2003 - MHDM03; Falcke et al. 2004 - FKM04). In X-ray binaries, it has become well established also that the radio emission is suppressed when the accretion disk enters into a high/soft state (e.g. Tananbaum et al. 1972; Fender et al. $1999)$ - i.e. when the X-ray spectrum is well fit by a geometrically thin disk model (e.g. Shakura \& Sunyaev 1973) rather than by a hard power law component. More recently, this has also been shown to be likely in AGN with soft X-ray spectra and luminosities of a few percent of the Eddington limit (Maccarone et al. 2003 - MGF03). Theoretical models also predict suppression of the radio emission in the soft state large scale height magnetic fields are typically needed to extract power from either the accretion disk or the black hole's spin into a relativistic jet (e.g. Livio et al. 1999; Meier 2001).

On the other hand, the velocity of a matter jet (i.e. one not dominated by Poynting flux) should be approximately the escape velocity of matter from the orbital radius where the jet is ejected. The lowest luminosity states, in which the geometrically thick part of the accretion disk is thought to extend the furthest away from the compact object (e.g. Esin et al. 1997), should thus have the lowest jet velocities (e.g. Meier 1999). Observations of the evolution of jet properties in Galactic X-ray transients seem to indicate at least that the jet velocities seen when sources are at high X-ray luminosities are higher than the jet velocities seen at low X-ray luminosities (Fender et al. 2004). It is thus possible, and perhaps quite reasonable, that a substantial fraction of the jet suppression in high/soft state systems is not because the jet is intrinsically weaker, but rather because the jet velocity is quite high and the jet will be Doppler de-boosted, except on lines of sight very close to our own. Such jets, when pointed at us, might be expected to have rather remarkable properties, and to allow us to probe new physics regimes not allowed by more normal jets, so searches for them are quite important.

One such source (RGB J0044+193) had been argued to show evidence for strong variability, as it was seen at a level of $24 \mathrm{mJy}$ at $5 \mathrm{GHz}$ in an update to the 1987 Green Bank (87GB) survey (see Gregory \& Condon 1991 for discussion of the survey; see also the updates to the catalog of Neumann et al. 1994; Brinkmann et al. 1995), undetected at $1.4 \mathrm{GHz}$ in the 1992 NRAO VLA Sky Survey (NVSS - see Condon et al. 1998) observations (which implied an upper limit of about $2.5 \mathrm{mJy}$ ), and detected again in a Very Large Array (VLA) follow-up survey of the 1987 Green Bank sources, with a flux density level of $7 \mathrm{mJy}$ at $5 \mathrm{GHz}$ (Laurent-Muehleisen et al. 1997). We direct the reader to Siebert et al. (1999) for a summary of the past radio observations and arguments that these observations 
imply a variable source. In this research note, we will present a re-analysis of the VLA follow-up observations, showing a source at a flux density level of about $0.8 \mathrm{mJy}$, as well as data from new Westerbork observations showing the same source at a flux density level of about $0.7 \mathrm{mJy}$. We also show that there is marginal evidence for the source in the NVSS data (albeit at a flux density level below the threshold for listing in the NVSS catalogs), and that this flux density level is consistent with our detection of the source at $2.0 \mathrm{mJy}$ at $1.4 \mathrm{GHz}$ in new Westerbork data, and note that the source was undetected in several observations with the Ryle telescope at $15 \mathrm{GHz}$, consistent with the extrapolation of the radio spectrum obtained with Westerbork and the hypothesis of little or no radio variability. We thus consider the possibility that the $87 \mathrm{~GB}$ detection was spurious and that this object is not strongly variable and has never been radio loud.

\section{Data used, analysis procedure, and results}

The dates, frequencies and flux densities of the observations used in this paper are shown in Table 1. Below appears a description of the reduction procedures. We note that RGB J0044+193 and the nearby background sources appear to be point-like within the instrumental angular resolutions, so we assume the flux density in mJy to be equal to the peak flux density in $\mathrm{mJy} /$ beam for each source.

\subsection{Westerbork observatory}

We observed RGB J0044+193 with the Westerbork Radio Synthesis Telescope for $24 \mathrm{~h}$ on source $(12 \mathrm{~h}$ at $1.4 \mathrm{GHz}$ and $12 \mathrm{~h}$ at $5 \mathrm{GHz}$ ), plus two hours of calibration time, on November 29 and 30, 2003. The data was analyzed using the MIRIAD package (Sault et al. 1995). The source was detected at both frequencies, with a flux density level of $2.0 \mathrm{mJy}$ at $1.4 \mathrm{GHz}$, and $0.8 \mathrm{mJy}$ at $5 \mathrm{GHz}$. The noise levels were $10 \mu \mathrm{Jy}$ at $1.4 \mathrm{GHz}$ and $70 \mu \mathrm{Jy}$ at $5 \mathrm{GHz}$. Allowing for some mild possible errors in absolute flux density calibration, we estimate that the errors should be no more than about $0.1 \mathrm{mJy}$ in both bands. The implied spectral index from these two data points is $\alpha=-0.7$, where the flux density, $f_{v}$ is expressed as a power law function of the frequency such that $f_{v} \propto v^{\alpha}$. In Fig. 1, we present the $5 \mathrm{GHz}$ Westerbork image.

\subsection{VLA}

The VLA has made three observations of fields including RGB J0044+193 - two at $5 \mathrm{GHz}$ and one at $1.4 \mathrm{GHz}$. One of the $5 \mathrm{GHz}$ observations was quite short ( $90 \mathrm{~s}$ ) and contained no suitable phase calibrator (the closest phase calibrator on the sky was 45 degrees away), so we have not analyzed it. The observation at $1.4 \mathrm{GHz}$ is the NVSS observation; no source appears in the NVSS catalog (Condon et al. 1998). The final observation was from the survey of Laurent-Muehleisen et al. (1997), which claimed a detection of this object at $7 \mathrm{mJy}$. We have reanalyzed this observation, calibrating and imaging the data using standard procedures and the NRAO AIPS data reduction package. 3C 286 was used as the primary calibrator,
Table 1. The radio data used in this paper - the observatory used, the frequency at which the data was taken, the date of the observations, the measured flux density, and a comment flag. Comments: (1) the Green Bank point may be spurious for reasons discussed in the text. (2) This flux density was reported to be $7 \mathrm{mJy}$, but we argue in the text that this report was erroneous, and (3) this is the data point from the NVSS; the images show a possible source at $1.5 \pm 0.5 \mathrm{mJy}$.

\begin{tabular}{|c|c|c|c|c|}
\hline Obs. & Freq. & Date & $\begin{array}{l}\text { Flux } \\
\text { dens. }\end{array}$ & Notes \\
\hline GB & $5 \mathrm{GHz}$ & 1987 & $24 \mathrm{mJy}$ & (1) \\
\hline VLA & $5 \mathrm{GHz}$ & 1994 Sep. 16 & $0.8 \mathrm{mJy}$ & (2) \\
\hline WSRT & $5 \mathrm{GHz}$ & 2003 Nov. $29-30$ & $0.8 \mathrm{mJy}$ & \\
\hline WSRT & $1.4 \mathrm{GHz}$ & 2003 Nov. $29-30$ & $2.0 \mathrm{mJy}$ & \\
\hline VLA & $1.4 \mathrm{GHz}$ & 1991 Feb. 1 & non-detection & (3) \\
\hline Ryle & $15 \mathrm{GHz}$ & 2003 & $0.31 \mathrm{mJy}$ & \\
\hline
\end{tabular}

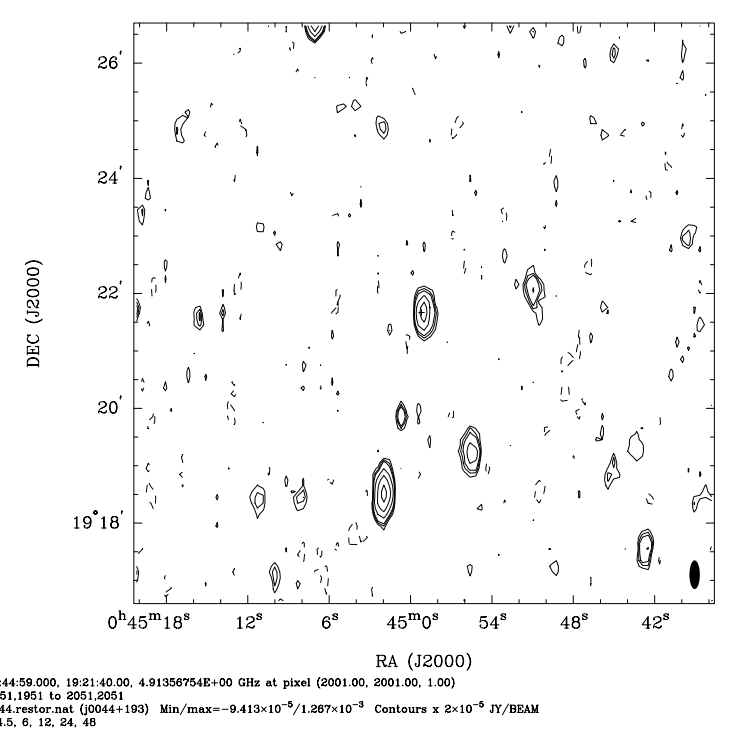

Fig. 1. The Westerbork observations at $5 \mathrm{GHz}$. The VLA map looks essentially the same, except that it has higher angular resolution.

and J0042 +233 ( $4^{\circ}$ from the target) as the secondary calibrator. Imaging was carried out using natural weighting in order to maximise the signal-to-noise ratio. One round of phase-only self-calibration was carried out before making the final image. It was necessary to image a large field $\left(13^{\prime} \times 13^{\prime}\right)$, since it was found that RGB J 0045+193 lay on the sidelobe of a 1.2-mJy source $5.5^{\prime}$ to the northeast of the target, and it was important to be able to accurately remove the sidelobes in order to measure the target source flux density.

We detect RGB J0044+193 at a flux density level of $765 \pm$ $91 \mu \mathrm{Jy}$. It is likely that the report of a $7 \mathrm{mJy}$ flux density in previous work was the result of a typographical or other clerical error (S. Laurent-Muehleisen, private communication). Two other sources in the field appear with consistent flux densities in the both of 5-GHz observations with the WSRT and our re-reduction of the VLA data, providing confidence in the measurements of J0044+193. 


\subsection{Ryle telescope}

The Ryle Telescope observed RGB J0044+193 at $15 \mathrm{GHz}$ 24 times from May through August of 2003 (see Pooley \& Fender 1997 for a description of the data reduction procedures). No detections were made. The averaged flux density at the source position is about $0.31 \pm 0.15 \mathrm{mJy}$, consistent with the $\alpha=-0.7$ power law spectrum measured with Westerbork.

\section{Comparison with past results and discussion}

Visual inspection of the publicly available NVSS FITS images shows a $1.5 \mathrm{mJy}$ source at $1.4 \mathrm{GHz}$ at exactly the position of RGB J0044+193. While this is not a convincing detection taken on its own (it is about $3 \sigma$ above the noise), it is within $1 \sigma$ of the flux density of the source at $1.4 \mathrm{GHz}$ found with Westerbork, indicating that large scale variability at $1.4 \mathrm{GHz}$ was unlikely over the 11 -year period between the NVSS data point and the Westerbork observation. The flux densities measured by the VLA in 1994 and by Westerbork in 2003 at $5 \mathrm{GHz}$ are also consistent with one another at the $1 \sigma$ level, and the $3 \sigma$ upper limit on the variability level is only about $40 \%$. It seems rather unlikely that source would vary by a factor of 30 between 1987 and 1994, while showing no evidence for variability between 1994 and 2003. On the other hand, little is known about jets from soft state accretion disks, so these systems might exhibit brief transient episodes.

Apart from this evidence for constancy of the source over an 11-year baseline, there is also some evidence that the 1987 Green Bank survey detection of RGB J0044+193 was spurious. First, we note that this object was not in either the original 87GB catalog (Gregory \& Condon 1991) or the followup of that catalog using a second epoch of data (Gregory et al. 1996). The nearest catalogued source to the position of RGB J0044+193 is a $34 \mathrm{mJy}$ source about 11 arcmin away, with a positional uncertainty of about 20 arcsec, and the sensitivity limit reported by Gregory et al. (1996) is about $22 \mathrm{mJy}$ at a declination of +19 degrees. The Green Bank catalog was also examined by Neumann et al. (1994) who produced an additional catalog of sources in that data, but RGB J0044+193 did not appear in their published catalog; the first publication of the radio flux density of RGB J0044+193 appeared in Brinkmann et al. (1995), who found a flux density value of $24 \mathrm{mJy}$ with an error in the 14-23\% range. We have communicated directly with W. Brinkmann and he is confident that the source detection was real, but is unable to recall so long after the fact why it appeared in only one of these two papers. However, this detection corresponds to a 4 to $7 \sigma$ detection level. Since the $87 \mathrm{~GB}$ survey covered 20000 square degrees with about 2 arcmin angular resolution, some spurious $4 \sigma$ detections are likely; the exact amount is difficult to quantify because the sensitivity limit is due to source confusion rather than Gaussian noise.

The basis for the previous claims that RGB J0044+193 was a radio loud AGN was the flux density ratio of 31 between $5 \mathrm{GHz}$ and the optical magnitude (Siebert et al. 1999), where the cutoff between radio quiet and radio loud is defined to be a ratio of 10 (Kellermann et al. 1989). The reduction of the maximum radio flux density by a factor of 30 implied by these results yields a ratio of radio-to-optical flux of about 1 . We are thus left with two possibilities - that the source was flaring when it was observed during the 1987 Green Bank survey, and hence that its radio variability is among the very strongest for all AGN, or that the detection in the 1987 Green Bank data was spurious, and hence that this source was never radio loud and shows no signs of radio variability. While we consider the latter more likely, we believe that the former cannot be excluded at the present time. Future radio monitoring of this source would be quite useful; we also note that this source should be brighter than predicted sensitivity limits of the Low Frequency Array (Röttgering 2003), so in the near future, detection of flaring activity would not require a dedicated monitoring program.

Acknowledgements. We are grateful to Elena Gallo and Dave Meier for useful discussions about jets from soft state systems; Sally Laurent-Muehleisen for useful discussions about the VLA followup survey of the 1987 Green Bank sources and for providing data to us, Margo Aller for providing data about the most variable AGN in the University of Michigan monitoring source lists, and to the Westerbork Radio Observatory staff for flexible scheduling. This work uses archival data from the VLA, part of NRAO. The NRAO is a facility of the National Science Foundation operated under cooperative agreement by Associated Universities, Inc. The Westerbork Synthesis Radio Telescope is operated by the ASTRON (Netherlands Foundation for Research in Astronomy) with support from the Netherlands Foundation for Scientific Research (NWO). The Ryle Telescope is supported by PPARC.

\section{References}

Boroson, T. A. 2002, ApJ, 565, 78

Brinkmann, W., Siebert, J., Reich, W., et al. 1995, A\&AS, 109, 147

Condon, J. J., Cotton, W. D., Greisen, E. W., et al. 1998, AJ, 115, 1693

Esin, A. A., McClintock, J. E., \& Narayan, R. 1997, ApJ, 489, 865

Falcke, H., Körding, E., \& Markoff, S. 2004, A\&A, 414, 895

Fender, R., Belloni, T., \& Gallo, E. 2004, MNRAS, 355, 1105

Fender, R. P., Corbel, S., Tzioumis, T., et al. 1999, ApJ, 519, L165

Gallo, E., Fender, R. P., \& Pooley, G. G. 2003, MNRAS, 344, 60

Gregory, P. C., \& Condon, J. J. 1991, ApJS, 75, 1011

Gregory, P. C., Scott, W. K., Douglas, K., \& Condon, J. J. 1996, ApJS, 103,427

Kellermann, K. I., Sramek, R., Schmidt, M., Shaffer, D. B., \& Green, R. 1989, AJ, 98, 1195

Laurent-Muehleisen, S. A., Kollgaard, R. I., Ryan, P. J., et al. 1997, A\&AS, 122, 235

Livio, M., Ogilvie, G. I., \& Pringle, J. E. 1999, ApJ, 512, 100

Maccarone, T. J., Gallo, E., \& Fender, R. 2003, MNRAS, 345, L19

Meier, D. L. 1999, ApJ, 522, 753

Meier, D. L. 2001, ApJ, 548, L9

Merloni, A., Heinz, S., \& Di Matteo, T. 2003, MNRAS, 345, 1057

Neumann, M., Reich, W., Fuerst, E., et al. 1994, A\&AS, 106, 303

Pooley, G. G., \& Fender, R. P. 1997, MNRAS, 292, 925

Röttgering, H. 2003, NewAR, 47, 405

Sault, R. J., Teuben, P. J., \& Wright, M. C. H. 1995, Astronomical Data Analysis Software and Systems IV, ed. R. A. Shaw, H. E. Payne, \& J. J. E. Hayes, ASP Conf. Ser., 77, 433

Shakura, N. I., \& Sunyaev, R. A. 1973, A\&A, 24, 337

Siebert, J., Leighly, K. M., Laurent-Muehleisen, S. A., et al. 1999, A\&A, 348, 678

Tananbaum, H., Gursky, H., Kellogg, E., Giacconi, R., \& Jones, C. 1972, ApJ, 177, L5 